\title{
Numerical solution of Schrodinger equation using compact finite differences method and the cubic spline functions
}

\author{
Behnam Sepehrian ${ }^{1, *}$, Marzieh Karimi Radpoor ${ }^{2,3}$ \\ ${ }^{1}$ Department of Mathematics, Faculty of Science, Arak University, Arak 38156-8-8349, Iran \\ ${ }^{2}$ Young Researchers Club, Hamedan Branch, Iran \\ 3 Islamic Azad University, Hamedan, Iran \\ *Corresponding author E-mail: b-sepehrian@araku.ac.ir
}

Copyright (C2014 Sepehrian and Radpoor. This is an open access article distributed under the Creative Commons Attribution License, which permits unrestricted use, distribution, and reproduction in any medium, provided the original work is properly cited.

\begin{abstract}
In this paper, a high-order method for solving the Schrodinger equation is introduced. We apply a compact finite difference approximation for discretizing spatial derivatives and we use the $C^{1}$-cubic spline collocation method for the time integration of the resulting linear system of ordinary differential equations. The proposed method has fourth-order accuracy in both space and time variables. We can obtain both pointwise approximations at the all mesh points and, a cubic spline solution in each space step by the method. Numerical results show that the method is an efficient technique for solving the one-dimensional Schrodinger equation.
\end{abstract}

Keywords: Compact finite difference, Cubic Spline functions, Numerical solution, Schrodinger equation.

\section{Introduction}

Consider the Schrodinger equation

$-i \frac{\partial u}{\partial t}(x, t)=\frac{\partial^{2} u}{\partial x^{2}}(x, t)+w(x) u(x, t), \quad(x, t) \in[a, b] \times[0, T]$,

with initial condition

$u(x, 0)=\varphi(x)$,

and the boundary conditions

$u(a, t)=\psi_{1}(t), \quad u(b, t)=\psi_{2}(t), \quad t \geq 0$.

In Eq. (1), $u(x, t)$ is the wave function in continuous domain and $w(x)$ is an arbitrary potential function and $i=\sqrt{-1}$. The Schrodinger equation (1) is a fundamental equation of physics for describing quantum mechanical behavior. It is often called the Schrodinger wave equation, and is a partial differential equation that describes how the wave function of a physical system evolves over time. According to Dehghan and Shokri [4] this equation appears 
in electromagnetic wave propagations, in underwater acoustics (paraxial approximation of the wave equations) or also in optic [9] and design of certain optoelectronic devices [5] as it models an electromagnetic wave equation in a two-dimensional weakly guiding structure. It has also found its application in various quantum dynamics calculations $[5,6]$. For these reasons, the construction of efficient numerical schemes for solving Scrodinger equation represents an important task.

This equation is of interest from the numerical point of view, because in general, analytical solutions are not available. Dehghan and Shokri developed a numerical scheme for two-dimensional Schrodinger equation using collocation and radial basis functions [4]. A compact finite difference method was used for solving two-dimensional Schrodinger equation using boundary value method by Mohebbi and Dehghan [11], which has fourth-order accurate in both time and space variables. However their method only gives pointwise approximations. Meshless local boundary integral equation method was developed in [3]. Finite difference schemes based on the second order discretization of spatial derivatives and first or second order discretization of time derivatives have been investigated in [2,16]. Abdur et al. in [1] proposed a Chebyshev spectral collocation method for two-dimensional Schrodinger equation. Kalita et al. in [7] introduced an implicit semi-discrete higher order compact scheme with an averaged time discretization for Schrodinger equation which is second-order accurate in time and fourth-order accurate in space.

Recently, because of their high accuracy, compactness and high resolution, the high-order compact difference schemes have seen increasing popularity in computational fluid dynamics [15], computational acoustics [8] and electromagnetic [14]. In [10] the scheme have been applied for numerical solution of heat and advection-diffusion equations.

The purpose of this paper is to propose a method for solving Eq. (1) using cubic spline functions. We use the compact finite difference approximation of fourth-order for discretizing spatial derivatives of Schrodinger equation and the $C^{1}$-cubic spline collocation method for the resulting linear system of ordinary differential equations. The method has fourth-order accuracy in both space and time components, and in each space step gives a closed form approximation for the solution. $C^{1}$-cubic spline collocation technique is an A-stable method and has fourth-order accuracy $[12,13]$.

The paper is organized as follows: In Section 2 we propose a compact finite difference scheme for Schrodinger equation. In Section 3, we present a method based on fourth-order discretization of spatial derivatives of Eq. (1) and $C^{1}$-cubic spline collocation technique for the time integration. In this section, the stability conditions for the introduced method are investigated. Numerical results that illustrate the efficiency of the proposed method are reported in Section 4.

\section{A fourth-order compact finite difference scheme}

The basic approach for high-order compact difference methods is to introduce the standard compact difference approximations to the differential equations and then by repeated differentiation and associated compact differencing, a new high-order compact scheme will be developed that incorporates the effect of the leading truncation error terms in the standard method [15]. In this section we state the fourth-order compact finite difference scheme for the spatial derivatives of Eq. (1).

Consider the following partial differential equation

$$
\frac{\partial^{2} u}{\partial x^{2}}=Q(x, t)
$$

Denote the central difference schemes of order two for second derivatives of $u$ as

$\delta_{x}^{2} u=\frac{\partial^{2} u}{\partial x^{2}}+\frac{h^{2}}{12} \frac{\partial^{4} u}{\partial x^{4}}+O\left(h^{4}\right)$.

By Eqs. (3) and (4) at each point $x_{r}$ we get

$\delta_{x}^{2} u_{r}-\tau_{r}=Q_{r}$

in which

$\tau_{r}=\frac{h^{2}}{12}\left(\frac{\partial^{4} u}{\partial x^{4}}\right)_{r}+O\left(h^{4}\right)$. 
Eq. (3) gives

$\left.\frac{\partial^{4} u}{\partial x^{4}}\right|_{x=x_{r}}=\left.\frac{\partial^{2} Q}{\partial x^{2}}\right|_{x=x_{r}}$.

By (5), (6) and (7) we get

$\delta_{x}^{2} u_{r}=\left(1+\frac{h^{2}}{12} \delta_{x}^{2}\right) Q_{r}+O\left(h^{4}\right)$

This relation is a fourth-order compact finite difference scheme for Eq. (3)(see also [11]).

\section{The proposed method}

For positive integers $n$ let $h=\frac{b-a}{n}$ denotes the step size of spatial derivatives and $\Delta t$ for step size of time variable $t$. We define

$$
\begin{aligned}
& x_{r}=r h \quad, \quad r=0,1, \cdots, n, \\
& t_{k}=k \Delta t \quad, \quad k=0,1, \cdots, N .
\end{aligned}
$$

Now, we rewrite (1) in the following form

$-i \frac{\partial u}{\partial t}(x, t)-w(x) u(x, t)=\frac{\partial^{2} u}{\partial x^{2}}(x, t)$.

By fourth-order scheme (8), Eq. (9) in each point $x_{r}$ gives

$i\left(1+\frac{h^{2}}{12} \delta_{x}^{2}\right) u_{r}^{\prime}(t)=-\delta_{x}^{2} u_{r}(t)-\left(1+\frac{h^{2}}{12} \delta_{x}^{2}\right) w_{r} u_{r}(t), \quad r=1,2, \ldots, n-1$

where $u_{r}(t) \approx u\left(x_{r}, t\right), w_{r}=w\left(x_{r}\right)$ and $u_{r}^{\prime}(t) \approx \frac{d}{d t} u\left(x_{r}, t\right)$. If we use $\delta_{x}^{2}\left(w_{r} u_{r}\right)=\left(\delta_{x}^{2} w_{r}\right) u_{r}+2\left(\delta_{x} w_{r}\right)\left(\delta_{x} u_{r}\right)+$ $w_{r}\left(\delta_{x}^{2} u_{r}\right)$ in which $\delta_{x} u_{r}$ and $\delta_{x}^{2} u_{r}$ are respectively the central difference schemes of order two for first and second derivatives of $u$ at $x=x_{r}$, and put $\delta_{x} w=H^{1}$ and $\delta_{x}^{2} w=H^{2}$ then we can rewrite the right-hand side of (10) as follows

$$
\begin{aligned}
& -\frac{1}{h^{2}}\left(u_{r-1}-2 u_{r}+u_{r+1}\right)-w_{r} u_{r}(t)-\frac{h^{2}}{12}\left(\delta_{x}^{2} w_{r}\right) u_{r}(t)-\frac{2 h^{2}}{12}\left(\delta_{x} w_{r}\right)\left(\delta_{x} u_{r}\right)-\frac{h^{2}}{12} w_{r}\left(\delta_{x}^{2} u_{r}\right) \\
& =-\frac{1}{h^{2}}\left(u_{r-1}-2 u_{r}+u_{r+1}\right)-w_{r} u_{r}(t)-\frac{h^{2}}{12} H_{r}^{2} u_{r}(t)-\frac{h^{2}}{6} H_{r}^{1}\left(\frac{u_{r+1}-u_{r-1}}{2 h}\right)-\frac{h^{2}}{12} w_{r}\left(\frac{u_{r-1}-2 u_{r}+u_{r+1}}{h^{2}}\right) \\
& =u_{r-1}\left(-\frac{1}{h^{2}}+\frac{h H_{r}^{1}}{12}-\frac{w_{r}}{12}\right)+u_{r}\left(\frac{2}{h^{2}}-\frac{5}{6} w_{r}-\frac{h^{2}}{12} H_{r}^{2}\right)+u_{r+1}\left(-\frac{1}{h^{2}}-\frac{h H_{r}^{1}}{12}-\frac{w_{r}}{12}\right),
\end{aligned}
$$

and therefore we can rewrite Eq. (10) as follows

$$
\begin{aligned}
& i\left[\frac{1}{2} u_{r-1}^{\prime}+5 u_{r}^{\prime}+\frac{1}{2} u_{r+1}^{\prime}\right]=u_{r-1}\left(-\frac{6}{h^{2}}+\frac{h H_{r}^{1}}{2}-\frac{w_{r}}{2}\right)+u_{r}\left(\frac{12}{h^{2}}-5 w_{r}-\frac{h^{2}}{2} H_{r}^{2}\right)+ \\
& u_{r+1}\left(-\frac{6}{h^{2}}-\frac{h H_{r}^{1}}{2}-\frac{w_{r}}{2}\right) .
\end{aligned}
$$

By Eq. (11) a system of ordinary differential equations is obtained as

$A u^{\prime}(t)=B u(t)+C(t)$, 
in which

$$
\begin{aligned}
& u(t)=\left[u_{1}(t), \ldots, u_{n-1}(t)\right]^{T}, \\
& A=\operatorname{trid}\left[\frac{i}{2}, 5 i, \frac{i}{2}\right]_{(n-1)}, \\
& B=\operatorname{trid}\left[-\frac{6}{h^{2}}+\frac{h H_{r}^{1}}{2}-\frac{w_{r}}{2}, \frac{12}{h^{2}}-5 w_{r}-\frac{h^{2}}{2} H_{r}^{2},-\frac{6}{h^{2}}-\frac{h H_{r}^{1}}{2}-\frac{w_{r}}{2}\right]_{(n-1)}, \quad r=1,2, \ldots, n-1 \\
& C(t)=\left[\left(-\frac{6}{h^{2}}+\frac{h H_{1}^{1}}{2}-\frac{w_{1}}{2}\right) u_{0}(t)-\frac{i}{2} u_{0}^{\prime}(t), 0, \ldots, 0,\left(-\frac{6}{h^{2}}-\frac{h H_{n-1}^{1}}{2}-\frac{w_{n-1}}{2}\right) u_{n}(t)-\frac{i}{2} u_{n}^{\prime}(t)\right]^{T},
\end{aligned}
$$

and $\operatorname{trid}\left[a_{1}, a_{2}, a_{3}\right]_{n-1}$ denotes the $(n-1) \times(n-1)$ tridiagonal matrix whose each row contains the values $a_{1}, a_{2}$ and $a_{3}$ on its subdiagonal, diagonal and superdiagonal, respectively. Also in (14), $u_{0}(t), u^{\prime}{ }_{0}(t), u_{n}(t)$ and $u_{n}^{\prime}(t)$ are obtained from boundary conditions (2).

From (13), the matrix $A$ in (12) is strictly diagonally dominant and consequently $A$ is invertible. Define $M=A^{-1} B$ and $P=A^{-1}$ then (12) can be written as follows

$u^{\prime}(t)=M u(t)+P C(t)=F(t, u(t))$.

Let $U(t)$ be a vector that approximates $u(t)$ such that each of its component is a cubic spline function. Now, suppose that $U(t)$ satisfies (15) at collocation points $t_{j-1}, t_{j-\frac{1}{2}}$ and $t_{j}$ in the time interval $\left[t_{j-1}, t_{j}\right]$ i.e. $U^{\prime}\left(t_{l}\right)=F\left(t_{l}, U\left(t_{l}\right)\right), l=j-1, j-\frac{1}{2}, j$.

From $[12,13]$ we have the following relations

$U(t)=U^{j-1}+\Delta t T_{1}(m) U^{\prime j-1}+\Delta t T_{2}(m) U^{\prime j-\frac{1}{2}}+\Delta t T_{3}(m) U^{\prime j}, \quad t \in\left[t_{j-1}, t_{j}\right]$

where

$$
\begin{aligned}
& T_{1}(m)=m-\frac{3}{2} m^{2}+\frac{2}{3} m^{3}, \quad T_{2}(m)=2 m^{2}-\frac{4}{3} m^{3}, \\
& T_{3}(m)=-\frac{1}{2} m^{2}+\frac{2}{3} m^{3}, \quad t=t_{j-1}+m \Delta t, \quad m \in[0,1] .
\end{aligned}
$$

By (15) and (16) we can write

$$
\begin{aligned}
& U^{j}=U^{j-1}+\frac{\Delta t}{6}\left[M U^{j-1}+P C^{j-1}+4 M U^{j-\frac{1}{2}}+4 P C^{j-\frac{1}{2}}+M U^{j}+P C^{j}\right], \\
& U^{j-\frac{1}{2}}=U^{j-1}+\frac{\Delta t}{24}\left[5 M U^{j-1}+5 P C^{j-1}+8 M U^{j-\frac{1}{2}}+8 P C^{j-\frac{1}{2}}-M U^{j}-P C^{j}\right],
\end{aligned}
$$

in which $U^{j}=U\left(t_{j}\right), C^{j}=C\left(t_{j}\right), U^{\prime j}=U^{\prime}\left(t_{j}\right)$ and so on. After some manipulation (17) and (18) can be written as

$$
\left(I-\frac{\Delta t}{6} M\right) U^{j}=\left(I-\frac{\Delta t}{6} M\right) U^{j-1}+\frac{2 \Delta t}{3} M U^{j-\frac{1}{2}}+\frac{\Delta t}{6} P\left(C^{j-1}+4 C^{j-\frac{1}{2}}+C^{j}\right),
$$

and

$$
\left(I-\frac{\Delta t}{3} M\right) U^{j-\frac{1}{2}}=\left(I-\frac{5 \Delta t}{24} M\right) U^{j-1}-\frac{\Delta t}{24} M U^{j}+\frac{\Delta t}{24} P\left(5 C^{j-1}+8 C^{j-\frac{1}{2}}-C^{j}\right),
$$

respectively, where $I$ is the $(n-1) \times(n-1)$ identity matrix. Multiplying both sides of (19) and (20) by $\left(I-\frac{\Delta t}{3} M\right)$ and $\frac{2 \Delta t}{3} M$ respectively, and summing resulted equations give us

$$
\begin{aligned}
& \left(I-\frac{\Delta t}{2} M+\frac{\Delta t^{2}}{12} M^{2}\right) U^{j}=\left(I+\frac{\Delta t}{2} M+\frac{\Delta t^{2}}{12} M^{2}\right) U^{j-1}+\left(\frac{\Delta t}{6} P+\frac{\Delta t^{2}}{12} P M\right) C^{j-1}+\frac{2 \Delta t}{3} P C^{j-\frac{1}{2}} \\
& +\left(\frac{\Delta t}{6} P-\frac{\Delta t^{2}}{12} P M\right) C^{j} .
\end{aligned}
$$


The above relation is a linear system of $(n-1)$ equations. By solving it for $U^{j}, j=1,2, \ldots, N$, the discrete and then by using (15), (16) and (18) a cubic spline approximation of $u\left(x_{r}, t\right)$ in $\left[t_{j-1}, t_{j}\right]$ can be obtained. Note that by multiplying Eq. (21) in $A^{2}$ we can avoid of any matrix inverting.

To investigate the stability of the difference scheme (21), we consider the homogeneous boundary conditions case of it i.e.

$U^{j}=\Phi U^{j-1}, \quad j=1,2, \ldots, N$,

where the amplification matrix is given by

$\Phi=\left(I-\frac{\Delta t}{2} M+\frac{\Delta t^{2}}{12} M^{2}\right)^{-1}\left(I+\frac{\Delta t}{2} M+\frac{\Delta t^{2}}{12} M^{2}\right)$.

Let $\lambda \in C$ be an eigenvalue of $M$ and $z=\Delta t \lambda$. For unconditionally stability of the method it is necessary that the absolute values of the eigenvalues of amplification matrix $\Phi$ be less than one, i.e.

$\left|\frac{1+\frac{z}{2}+\frac{z^{2}}{12}}{1-\frac{z}{2}+\frac{z^{2}}{12}}\right|<1$

Let $z=a+b i, z_{1}=1+\frac{z}{2}+\frac{z^{2}}{12}$ and $z_{2}=1-\frac{z}{2}+\frac{z^{2}}{12}$. We have the following relations

$\left|z_{1}\right|^{2}=\left(1+\frac{a}{2}+\frac{a^{2}-b^{2}}{12}\right)^{2}+b^{2}\left(\frac{a}{6}+\frac{1}{2}\right)^{2}$,

and

$\left|z_{2}\right|^{2}=\left(1-\frac{a}{2}+\frac{a^{2}-b^{2}}{12}\right)^{2}+b^{2}\left(\frac{a}{6}-\frac{1}{2}\right)^{2}$.

As we see $\left|z_{1}\right|<\left|z_{2}\right|$ iff $a<0$ and $\left|z_{1}\right| \geq\left|z_{2}\right|$ if $a \geq 0$. Therefore the relation (23) holds iff $\operatorname{Re} \lambda<0$ and $z$ be in the left-half complex plane. So, in this case the method is A-stable.

Also, since the amplification matrix, $\Phi$, in $(22)$ is the $(2,2)$ Pade approximation of $e^{\Delta t M}$, so the method has fourth-order accuracy in time component.

\section{Numerical experiments}

We applied the method presented in this paper and solved two test problems. We performed our computations using maple 13 software.

\section{1. $\quad$ Test problem 1}

Consider Eq. (1) with $a=0, b=1$ and $w(x)=0$ and with the initial condition $\varphi(x)=\sin (x)$. The exact solution is

$u(x, t)=e^{-i t} \sin (x)$,

and the boundary conditions can be obtained easily from exact solution. In Table 1, the maximum errors of pointwise approximations and the experimental convergence orders obtained for Test problem 1 with different values of $h$ and $\Delta t$ at $T=1$ are presented. The followings are used for the maximum error at the time $T=1$ and the experimental convergence order $(C-$ order $)$.

$\|e\|_{l^{\infty}}=\max _{1 \leq i \leq n-1}\left\{\left|u_{\text {exact }}\left(x_{i}, 1\right)-u_{\text {approx. }}\left(x_{i}, 1\right)\right|\right\}$,

and

$C-$ order $=\log _{2}\left(\|e(2 h, 2 \Delta t)\|_{l^{\infty}} /\|e(h, \Delta t)\|_{l_{\infty}}\right)$,

in which $\|e(h, \Delta t)\|_{l^{\infty}}$ means the error $\|e\|_{l^{\infty}}$ computed with mesh sizes $h$ and $\Delta t$. 
Table 1: Maximum errors and experimental convergence orders obtained for test problem 1 at $T=1$.

\begin{tabular}{ccccc}
\hline Mesh sizes & \multicolumn{2}{c}{ Real part } & \multicolumn{2}{c}{ Imaginary part } \\
\hline$h=\Delta t$ & $\|e\|_{l^{\infty}}$ & $C$ - order & $\|e\|_{l^{\infty}}$ & $C$-order \\
\hline $1 / 5$ & $2.739 \times 10^{-6}$ & - & $2.832 \times 10^{-6}$ & - \\
$1 / 10$ & $1.836 \times 10^{-7}$ & 3.90 & $2.422 \times 10^{-7}$ & 3.55 \\
$1 / 20$ & $1.157 \times 10^{-8}$ & 3.99 & $1.279 \times 10^{-8}$ & 4.24 \\
$1 / 40$ & $7.355 \times 10^{-10}$ & 3.98 & $9.803 \times 10^{-10}$ & 3.70 \\
$1 / 80$ & $5.558 \times 10^{-11}$ & 3.73 & $6.113 \times 10^{-11}$ & 4.00 \\
\hline
\end{tabular}

Table 2: Maximum errors and experimental convergence orders obtained for test problem 2 at $T=1$.

\begin{tabular}{ccccc}
\hline Mesh sizes & \multicolumn{2}{c}{ Real part } & \multicolumn{2}{c}{ Imaginary part } \\
\hline$h=\Delta t$ & $\|e\|_{l^{\infty}}$ & $C$-order & $\|e\|_{l^{\infty}}$ & $C-$ order \\
\hline $1 / 5$ & $2.318 \times 10^{-4}$ & - & $7.018 \times 10^{-4}$ & - \\
$1 / 10$ & $4.547 \times 10^{-5}$ & 2.35 & $2.980 \times 10^{-5}$ & 4.56 \\
$1 / 20$ & $6.530 \times 10^{-6}$ & 2.80 & $4.314 \times 10^{-6}$ & 2.79 \\
$1 / 40$ & $1.038 \times 10^{-6}$ & 2.65 & $7.805 \times 10^{-7}$ & 2.47 \\
$1 / 80$ & $1.209 \times 10^{-7}$ & 3.10 & $7.513 \times 10^{-8}$ & 3.38 \\
\hline
\end{tabular}

\section{2. $\quad$ Test problem 2}

Consider

$$
\begin{aligned}
& -i \frac{\partial u}{\partial t}(x, t)=\frac{\partial^{2} u}{\partial x^{2}}(x, t)+\left(1-\frac{2}{x^{2}}\right) u(x, t), \quad(x, t) \in[0,1] \times[0, T], \\
& u(x, 0)=x^{2}, \\
& u(0, t)=0, \quad u(1, t)=e^{i t}, \quad t \geq 0 .
\end{aligned}
$$

The exact solution is $u(x, t)=x^{2} e^{i t}$. In Table 2 , the maximum errors and the experimental convergence orders resulted for pointwise solutions of Test problem 2 at $T=1$ are given.

\section{Conclusion}

In this paper, we proposed a class of new finite difference schemes, for solving the one-dimensional Schrodinger equations. We applied a compact finite difference scheme to approximate the spatial derivative, and the $C^{1}$-cubic spline collocation technique for the time component to solve the Schrodinger equations. The presented method can gives us both pointwise approximations in all mesh points, and a cubic spline solution in each space step. Also, the method has fourth-order accuracy in both space and time variables and is suitable for long time interval problems. The numerical results confirm the validity of the technique.

\section{References}

[1] R. Abdur, A.I.B.MD. Ismail, Numerical studies on two-dimensional schrodinger equation by chebyshev spectral collocation method, U.P.B. Sci. Bull. (2011) 1223-1227.

[2] M. Dehghan, Finite difference procedures for solving a problem arising in modeling and design of certain optoelectronic devices, Math. Comput. Simul. 71 (2006) 16-30.

[3] M. Dehghan, D. Mirzaei, Numerical solution to the unsteady two-dimensional Schrodinger equation using meshless local boundary integral equation method, Int. J. Numer. Math. Engng. 76 (2008) 501-520.

[4] M. Dehghan, A. Shokri, A numerical method for two-dimensional Schrodinger equation using collocation and radial basis functions, Comput. Math. Appl. 54 (2007) 136-146.

[5] F.Y. Hajj, Solution of the Schrodinger equation in two and three dimensions, J. Phys. B. 18 (1985) 1-11.

[6] L.Gr. Ixaru, Operations on oscillatory functions, Comput. Phys. Comm. 105 (1997) 1-9. 
[7] J.C. Kalita, P. Chhabra, S. Kumar, A semi-discrete higher order compact scheme for the unsteady two-dimensional Schrodinger equation, J. Comput. Appl. Math. 197 (2006) 141-149.

[8] S. Kim, Compact schemes for acoustics in the frequency domain, Math. Comput. Modeling, 37 (2003) $1335-1341$.

[9] Y.V. Kopylov, A.V. Popov, A.V. Vinogradov, Applications of the parabolic wave equations to X-ray diffraction optics, Optics Comm. 118 (1995) 619-636.

[10] A. Mohebbi, M. Dehghan, High-order compact solution of the one-dimensional heat and advection-diffusion equations, Applied Mathematical Modeling, 34 (2010) 3071-3084.

[11] A. Mohebbi, M. Dehghan, The use of compact boundary value method for the solution of two-dimensional Schrodinger equation, Journal of Computational and Applied Mathematics, 225 (2009) 124-134.

[12] S. Sallam, M. Naim Anwar, M. R. Abdel-Aziz, Unconditionally stable $C^{1}$-cubic spline collocation method for solving parabolic equations, International Journal of Computer Mathematics, (2004) 813-821.

[13] S. Sallam and M. Naim Anwar, Stabilized $C^{1}$-cubic spline collocation method for solving first-order ordinary initial value problems, International Journal of Computer Mathematics, (2000) 87-96.

[14] J.S. Shang, High-order compact difference schemes for time-dependent Maxwell equations, J. Comput. Phys. 153 (1999) 312-333.

[15] W.F. Spotz, High-order compact finite difference schemes for computational mechanics, Ph.D. Thesis, University of Texas at Austin, Austin, TX, 1995.

[16] M. Subasi, On the finite difference schemes for the numerical solution of two dimensional Schrodinger equation, Numer. Methods Partial Differential Equations, 18 (2002) 752-758. 